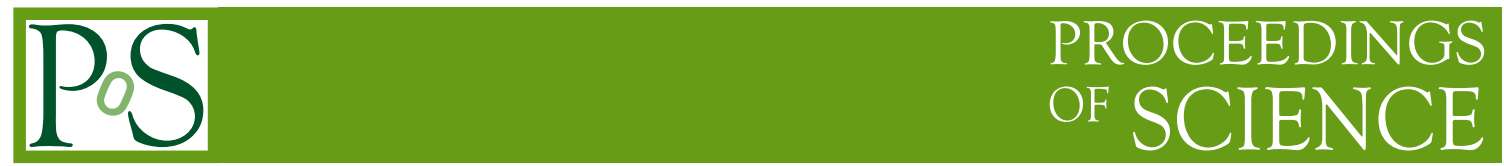

\title{
PoS Study of the process of scalar top pair production at ILC
}

\author{
Anna Skachkova* \\ JINR DNLP \\ E-mail: Anna.Skachkova@cern.ch
}

\begin{abstract}
We study pair production of scalar top quarks (stop, $\tilde{t}_{1}$ ) in polarized photon-photon collisions with the subsequent decay of the top squarks into $b$-quarks and charginos $\tilde{t}_{1} \rightarrow b \tilde{\chi}_{1}^{ \pm}$. We simulate this process by using PYTHIA6.4 for an electron beam energy $2 E_{\text {beam }}^{e}=\sqrt{s_{e e}}=1000 \mathrm{GeV}$. A set of criteria for physical variables is proposed which leads to a good separation of stop signal events from top quark pair production being the main background. These criteria allow us to reconstruct the mass of the top squark provided that the neutralino mass is known.
\end{abstract}

The XXth International Workshop High Energy Physics and Quantum Field Theory September 24 - October 1, 2011

Sochi, Russia

\footnotetext{
* Speaker.
} 


\section{Introduction.}

The scalar top quark, the bosonic partner of the top quark, is expected to be the lightest colored supersymmetric (SUSY) particle. $\tilde{t}_{L}$ and $\tilde{t}_{R}$, the supersymmetric partners of the left-handed and right-handed top quarks, mix and the resulting two mass eigenstates $\tilde{t}_{1}$ and $\tilde{t}_{2}$, can have a large mass splitting. It is even possible that the lighter eigenstate $\tilde{t}_{1}$ could be lighter than the top quark itself. Searches for top squarks were performed at LEP and Tevatron and will continue at LHC and ILC. At ILC it is planned to have the option of a photon collider (PLC), as originally planned for TESLA. This will be achieved by using backscattered photon beams by Compton scattering of laser photon beams with electron beams.

It has been stressed that the polarization effects in the interactions of backscattered laser photons provide additional opportunities for studying the properties of the produced particles. In the following we study the reaction

$$
\gamma+\gamma \rightarrow \tilde{t}_{1}+\tilde{\tilde{t}}_{1} .
$$

Among the possible $\tilde{t}_{1}$-decay channels within the MSSM (see [1] for details), we focus on the decay $\tilde{t}_{1} \rightarrow b \tilde{\chi}_{1}^{ \pm}$followed by the two-body chargino decay $\tilde{\chi}_{1}^{ \pm} \rightarrow \tilde{\chi}_{1}^{0} W^{ \pm}$, where one of the W's decays hadronically, $W \rightarrow q_{i} \bar{q}_{j}$, and the other one leptonically, $W \rightarrow \mu v_{\mu}{ }^{1}$. The final state of this signal process, shown in the left plot of Fig.1, contains two $b$-quarks and two quarks (originating from the decay of one $\mathrm{W}$ boson), a hard muon plus a neutrino (from the decay of the other $\mathrm{W}$ ) and two neutralinos:

$$
\gamma \gamma \rightarrow \tilde{t}_{1} \bar{t}_{1} \rightarrow b \bar{b} \tilde{\chi}_{1}^{+} \tilde{\chi}_{1}^{-} \rightarrow b \bar{b} W^{+} W^{-} \tilde{\chi}_{1}^{0} \tilde{\chi}_{1}^{0} \rightarrow b \bar{b} q_{i} \bar{q}_{j} \mu v_{\mu} \tilde{\chi}_{1}^{0} \tilde{\chi}_{1}^{0}
$$

The main background process is top quark pair production with the subsequent decay $t \rightarrow b W^{ \pm}$ (for W's we use the same decay channels as in the stop case):

$$
\gamma \gamma \rightarrow t \bar{t} \rightarrow b \bar{b} W^{+} W^{-} \rightarrow b \bar{b} q_{i} \bar{q}_{j} \mu v_{\mu}
$$

The only difference between the final states of stop and top production (shown in the right diagram of Fig.1) is that the stop pair production has two neutralinos which are undetectable. Thus, both processes have the same signature: two $b$-jets, two jets from $\mathrm{W}$ decay and a muon. In the following we show that the physical variables constructed out of the final state may us allow to reconstruct the scalar top quark mass. In the present paper we consider only top pair production as background.

We analyse the processes (1.2) (for $M_{t_{1}}=167.9 \mathrm{GeV}$ ) and (1.3) with the help of Monte Carlo samples of the corresponding events. Two programs PYTHIA6.4 and CIRCE2 were used. To simulate stop pair production process (1.1), we used the PYTHIA6.4 event generator in which the formula for the cross section of the stop pair production in $e^{+} e^{-}$annihilation was replaced by the formula for two scalar particles (s) production $\gamma \gamma \rightarrow s \bar{s}$ from [6], (see [7] for the NLO corrections and [8] for more details about differential cross sections), which takes into account various photon polarization states. The top background was also simulated with PYTHIA6.4. The program CIRCE2 was used to generate the momentum spectra of the backscattered photons involved in the process (1). The energy of the electron beams was chosen to be $E_{\text {beam }}^{e}=500 \mathrm{GeV}$ (i.e. the total $e^{-} e^{-}$energy is $\left.E_{e-e-}^{t o t}=\sqrt{s_{e e}}=1000 \mathrm{GeV}\right)$.

\footnotetext{
${ }^{1}$ The process $e^{+} e^{-} \rightarrow \tilde{t}_{1} \tilde{t}_{1}$ with the subsequent decay channels $\tilde{t}_{1} \rightarrow b \tilde{\chi}_{1}^{ \pm}$was studied in [月] and [阿.
} 
a)

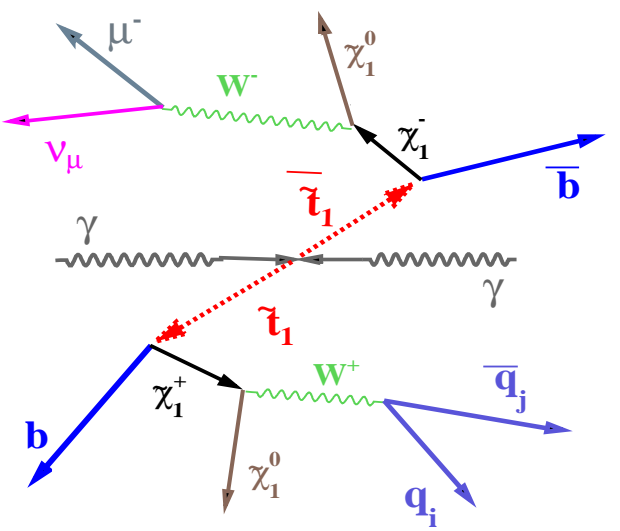

b)

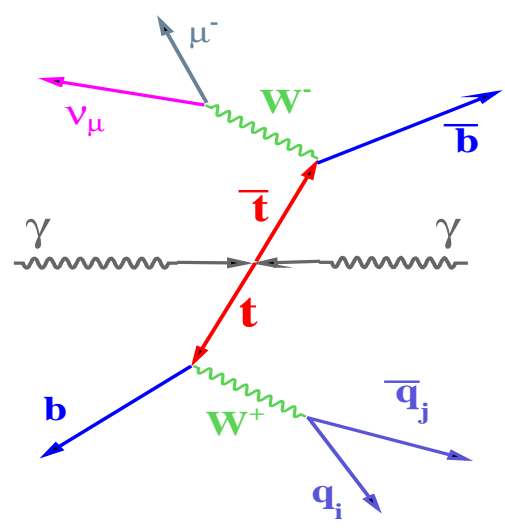

Figure 1: Left is the stop signal event diagram, Right is the top background diagram.

\section{Some global variables and signal-to-background ratio.}

In this Section we present some plots for the global variables distributions based on two samples of $2.5 \cdot 10^{4}$ stop pair production events generated by CIRCE2 and PYTHIA6.4. They were weighted by the photon-photon luminosity calculated with the help of CIRCE2 and for the corresponding polarizations. Analogous plots are also given for $1.0 \cdot 10^{5}$ generated background top events. The generation of all events, i.e. for the stop and top production, was done separately for both possible combinations of photon polarizations, i.e. for the same sign (" ++" and " - -") and for the opposite sign ("+-" and "-+") helicities.

To find the jets we use the subroutine PYCLUS of PYTHIA. The parameters of this jet finder are chosen such that the number of jets is exactly four.

In our work [ $₫$, [河] we have considered a set of global invariant variables, which can be useful for signal and background separation. Here we can present only the most important ones. Figure 2 shows the distributions of the total visible (detectable) energy in event $E_{v i s-t o t}$ in stop production (plots a) and b)) and in top production (plots $\mathbf{c}$ ) and d)). A cut on the total visible energy of approximately $E_{v i s-t o t}<250 \mathrm{GeV}$ would eliminate most of the top background while approximately $10 \%$ of the signal events are lost.

An even more efficient separation of the signal and the background can be obtained by using the invariant mass $M_{i n v}($ All jets $)$ of the system that contains all jets.

$$
M_{\text {inv }}(\text { All jets })=\sqrt{\left(\sum_{i=1}^{N^{j e t}} P_{j e t}^{i}\right)^{2}} .
$$

The corresponding distributions for the signal stop events (upper plots) and for the background top events (lower plots) are shown in Fig.3. It is seen that the application of the cut $M_{\text {inv }}$ (All jets) $\leq 180$ $\mathrm{GeV}$ leads to a practically complete separation of signal stop and top background events.

The variables $E_{v i s-t o t}$ and $M_{i n v}($ All jets $)$, by definition, include the total 4-momentum of all jets, defined as the vectorial sum of the 4-momenta of all jets. Therefore they do not suffer on energy redistribution between jets. Based on our results above, we will use the following three cuts to separate the signal and background events:

- there must be at least two $b$-jets in an event:

$$
N_{b-j e t s} \geq 2
$$


a)
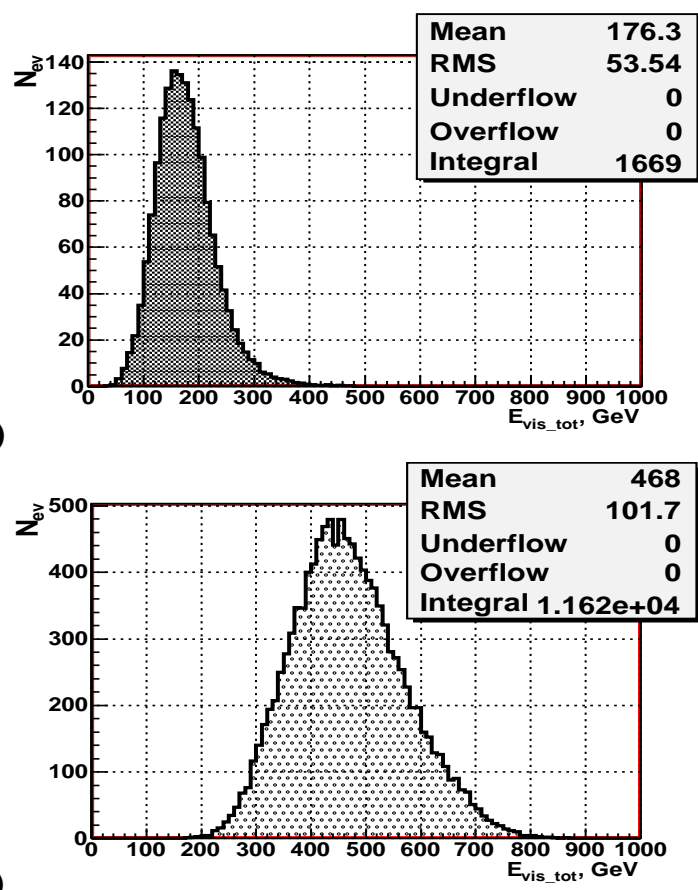

b)

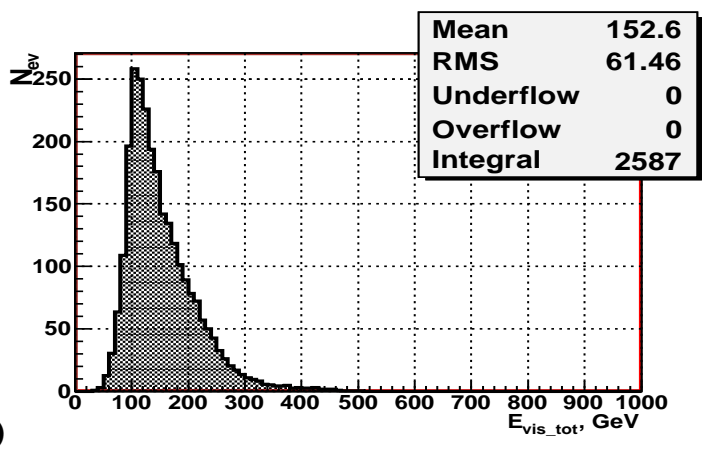

d)

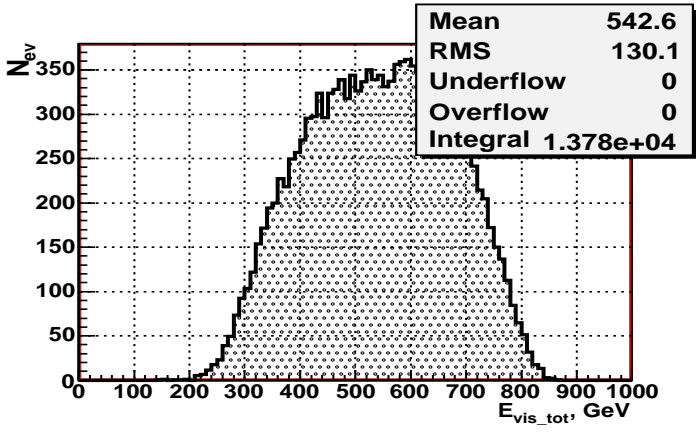

Figure 2: Total energy $E_{v i s-t o t}$ distribution. a) and b) are for stop pair production; c) and d) are for top pair production. a) and c) " +-" and "-+" polarizations, b) and d) "++" and"--" polarizations.

a)
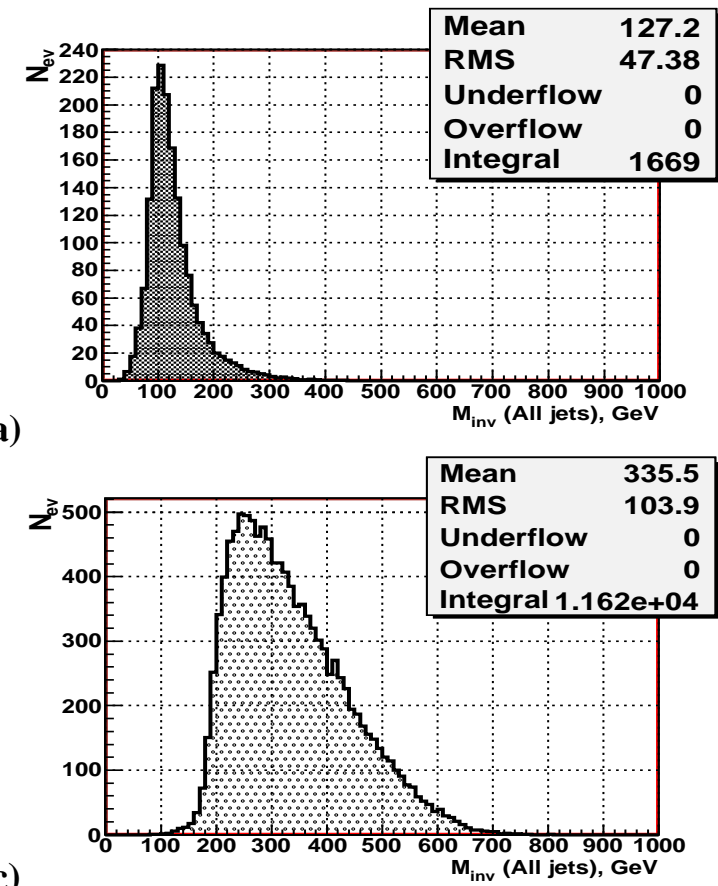

c)

Figure 3: Distribution of number of events versus the reconstructed invariant mass of all jets $M_{\text {inv }}($ All jets). a) and b) are for stop pair production; c) and d) are for top pair production. a) and c) " + -" and "-+" polarizations, b) and d) " + +" and "- -" polarizations.

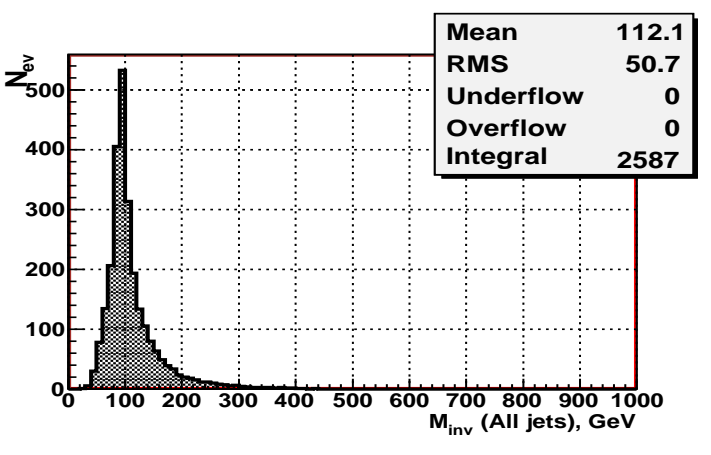

b)

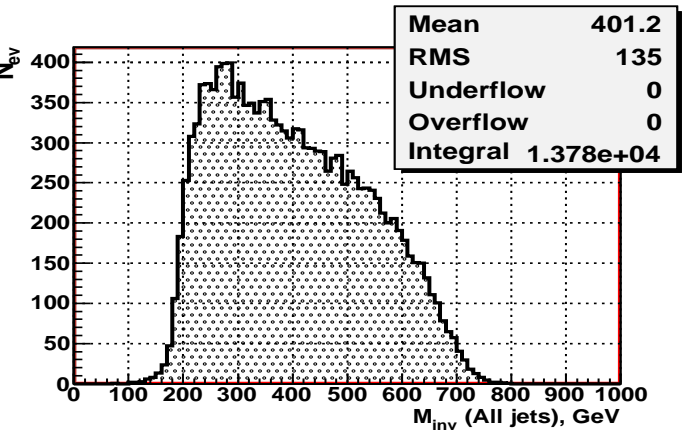

d) 
- the invariant mass of all jets must be less than $180 \mathrm{GeV}$ :

$$
M_{\text {inv }}(\text { All jets }) \leq 180 \mathrm{GeV}
$$

- the detected energy $E_{v i s-t o t}$ must be less than $250 \mathrm{GeV}$ :

$$
E_{v i s-t o t} \leq 250 \mathrm{GeV} \text {. }
$$

All the figures presented in this paper are obtained after applying the first cut in order to get the right picture of jets when the $b$-jets are clearly determined.

These three cuts for the case with $J=0$ enhanced state of the total angular momentum of the beams considered here, improve the signal-to-background ratio in the case of " + - and " - +" polarizations from $S / B=0.15$ to $S / B=59$, losing about $23.7 \%$ (from 1903 to 1453 ) of the signal stop events and reduction of background top events from $1.227 \cdot 10^{4}$ to 24 . In the case of " ++ " and "- -" polarizations an improvement of the signal-to-background ratio is from $S / B=0.22$ to $S / B=120$, with a loss about $27.6 \%$ (from 3233 to 2338 ) of the signal stop events and a reduction of the background top events from $1.441 \cdot 10^{4}$ to 19 .

\section{Determination of the scalar top quark mass.}

Another variable of interest is the invariant mass $M_{i n v}\left(b-j e t\right.$, Jet $\left.s_{W}\right)$ :

$$
M_{i n v}\left(b-j e t, J e t s_{W}\right) \equiv \sqrt{\left(P_{b-j e t}+P_{\text {Jets }}\right)^{2}},
$$

which is constructed as the modulus of the vectorial sum of the 4-momentum $P_{b-j e t}$ of the $b$-jet, plus the total 4-momentum of Jet $s_{W}$ system, i.e. non- $b$-jets stemming from the $\mathrm{W}$ decay $\left(P_{\text {Jets }}=\right.$ $P_{j e t 1_{W}}+P_{j e t 2 W}$, as there are only two jets allowed to be produced in $\mathrm{W}$ decay). This is only possible if one can discriminate between the $b$ - and $\bar{b}$-jets experimentally. Here we do not use any b-tagging procedure. The PYTHIA information about quark flavor is taken for choosing the $b$ - and $\bar{b}$-jets.

The distributions of the invariant masses of the " $b$-jet+Jet $s_{W}$ " system in the case of stop pair production are shown in plots a) and b) of Fig.4 for the two polarization combinations, as well as in the case of top pair production in plots $\mathbf{c})$ and $\mathbf{d})$. In the top case the invariant mass $M_{\text {inv }}(b-$ jet, 2 jet $s_{W}$ ) of the system composed of a $b$-jet and two jets from $\mathrm{W}$ decay should reproduce the mass of their parent top quark $M_{\text {top }}=170.9( \pm 1.8) \mathrm{GeV}$ (see Fig.1).

Note that, according to the stop decay chain (1.2), the right edge of the peak of the invariant mass distribution of the " $b$-jet+Jets $S_{W}$ " system corresponds to the mass difference $M_{\tilde{t}_{1}}-M_{\tilde{\chi}_{1}^{0}}$. The width between the grid dots in plot b) of Fig.4 is found to be about $7.4 \mathrm{GeV}$. Thus, we can estimate that the right edge of the peak of the $M_{i n v}\left(b\right.$-jet, Jet $\left.S_{W^{*}}\right)$ distribution lies a little higher than 85.2 $\mathrm{GeV}$. This allows one to reconstruct the input value of the stop mass $(167.9 \mathrm{GeV})$ by adding the mass of the neutralino to the value mentioned above. For this the mass of $\tilde{\chi}_{1}^{0}$ has to be known. More detailed description of the procedure of the stop-quark mass reconstruction see at [4], [5].

In conclusion, we can say that the $\gamma \gamma$ channel is very well suited for the study of stop pair production. 
a)

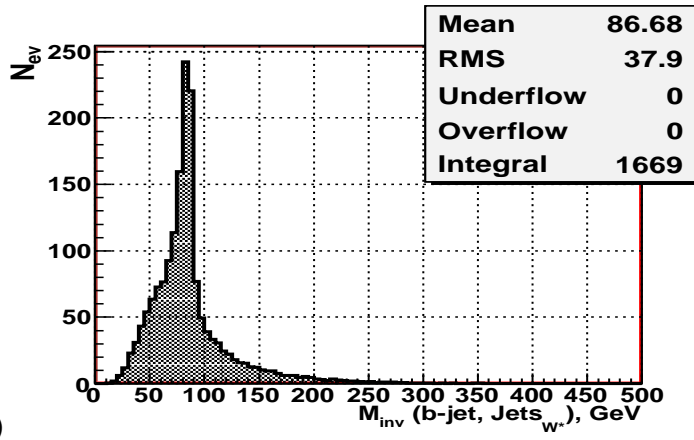

b)

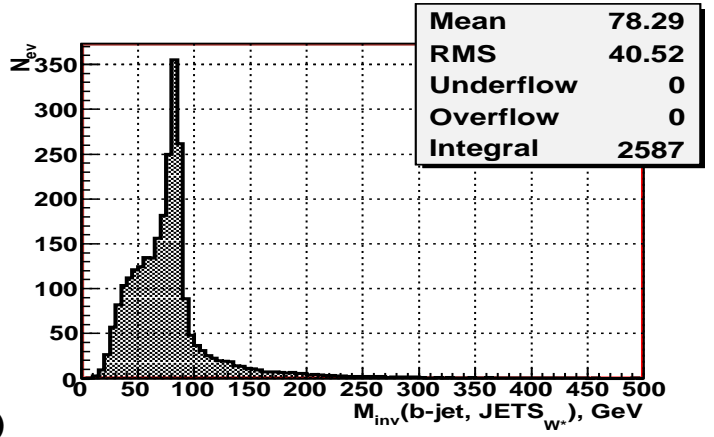

d)

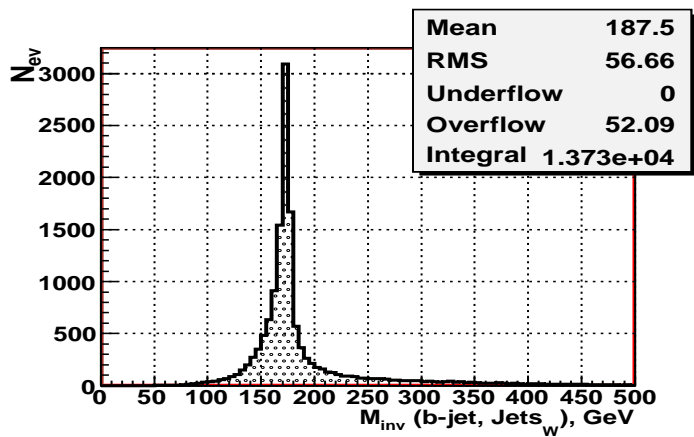

c)
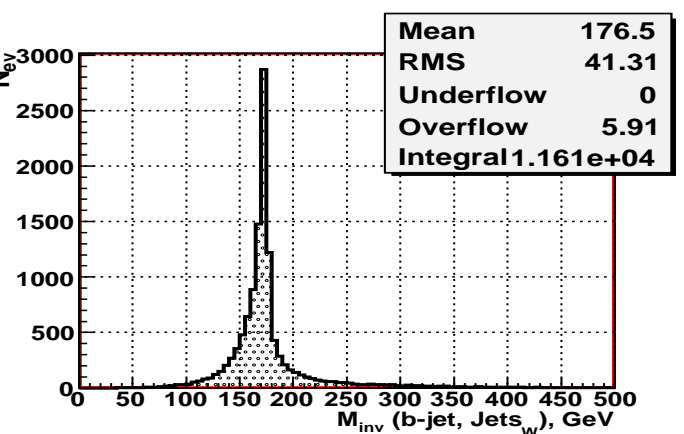

Figure 4: The spectra of the invariant masses $M_{i n v}\left(b-j e t\right.$, Jet $\left.s_{W}\right)$ obtained without use of cuts (2.3) and (2.4). a) and b) are for stop pair production; c) and d) are for top pair production. a) and c) " + -" and $"-+$ " polarizations, b) and $\mathbf{d}) "++"$ and $"-\ldots$ "polarizations.

\section{Acknowledgements.}

This work is supported by the JINR-BMBF project and by the "Fonds zur Förderung der wissenschaftlichen Forschung" (FWF) of Austria, project No.P18959-N16.

\section{References}

[1] A.Bartl, H.Eberl, S.Kraml, W.Majerotto and W.Porod, EPJ C2(2000)6; hep-ph/0002115.

[2] A.Bartl, K.Moenig, W.Majerotto, A.Skachkova, N.Skachkov, "On pair production of scalar top quarks in e+e- collisions at ILC and a possibility of their mass reconstruction",

Phys.Part.Nucl.Lett.6:181-189,2009.(No.3); arXiv:0906.3805 [hep-ph].

[3] A.Bartl, K.Moenig, W.Majerotto, A.Skachkova, N.Skachkov, "Pair production of scalar top quarks in e+e- collisions at ILC"; arXiv:0804.2125v3 [hep-ph].

[4] A.Bartl, K.Mönig, W.Majerotto, A.Skachkova, N.Skachkov, "Pair production of scalar top quarks in polarized photon-photon collisions at ILC"; arXiv:0804.1700v3 [hep-ph].

[5] A.Bartl, K.Mönig, W.Majerotto, A.Skachkova, N.Skachkov, "Scalar Top Quarks production in polarized photon-photon collisions at ILC”, Phys.Part.Nucl.Lett.9:53-76,2012.(No.171).

[6] I.F.Ginzburg, Nucl. Instrum. Meth., A355(1995)63.

[7] S. Berge, "Gluino and squark pair production at future linear colliders." DESY-THESIS-2003-048, Dec.2003, 106p.

[8] S. Berge, M. Klasen, Y. Umeda, Phys.Rev. D63:035003, 2001. 